\title{
Kinematic and Static Solutions for Beam Column with Nonlinear Springs Using the Extended Linear Matching Method
}

\author{
Bin Gao and Jun Wu \\ School of Urban Rail Transportation, Shanghai University of Engineering Science, Shanghai 201620, China \\ Correspondence should be addressed to Jun Wu; cvewujun@sues.edu.cn
}

Received 6 May 2021; Accepted 9 June 2021; Published 21 June 2021

Academic Editor: Kaiming Bi

Copyright (C) 2021 Bin Gao and Jun Wu. This is an open access article distributed under the Creative Commons Attribution License, which permits unrestricted use, distribution, and reproduction in any medium, provided the original work is properly cited.

\begin{abstract}
In this paper, the kinematic and static solutions for solving the static response of the beam column with nonlinear springs are presented by adopting the extended linear matching method (LMM). The extended LMM can be used to predict the displacement response of the beam-column system consisting of perfectly plastic and strain-softening materials. It is found that the kinematic solution generated by the extended LMM demonstrates a monotonic decrease for perfect plastic materials with certain restrictions on the yield surface. The potential energy of the system is proved to decrease with iterations for both perfect plastic and strainsoftening materials if the loading multiplier remains constant. The extended LMM method is then applied to analyse the response of the pile system in a 3-leg offshore platform. An incremental procedure is recommended to determine the peak load for the soil exhibiting strain-softening. A displacement-control approach is used with the loading multiplier obtained from the variation of the potential energy. Good convergence of the method is obtained.
\end{abstract}

\section{Introduction}

Classical limit analysis has been conducted extensively in the engineering due to its sound theoretical fundamental and practicability since 1950s. The upper and lower bound theorems provide the basis for bracketing the plastic limit load of a structural system that consists of the perfectly plastic materials subjected to small strain [1-5]. An alternative for implementing limit analysis is the linear matching method (LMM) [6, 7]. The LMM is a class of programming methods based on elastic solutions with spatially distributed material stiffness. In the LMM, finite element (FE) methods are usually adopted to find solutions that satisfy equilibrium and compatibility under the given boundary conditions on the assumption of the linear-elastic material. The plastic behavior of the certain portion of the system is simulated by the sequential adjustment of the corresponding stiffness iteratively. For the system consisting of perfectly plastic materials without geometric nonlinearities, Ponter et al. [6] demonstrated that the displacement field generated from an elastic solution can be treated as a plastic displacement field in the limiting case. Therefore, the upper and lower bound solutions can be obtained simultaneously through the LMM, and the exact solution associated with the displacement field allowed by the FE formation is then guaranteed by the convergence of the upper and lower bound solutions with certain restrictions on the yield surface (e.g., an incompressible material under the von Mises criterion).

For materials exhibiting strain-softening, the fundamental assumptions of limit analysis are violated. Hence, the analysis in general has to be conducted in an incremental way in order to determine the peak load since the plastic response of a material depends both on the accumulated plastic strains and the strain history. The computational issues associated with strain-softening materials are briefly discussed by Barrera et al. [8] from the material constitutive relation aspect and by Zienkiewicz and Taylor [9] from the numerical calculation aspect. For a specific class of proportional loading that loads can be expressed by a load multiplier (e.g., the pushover analysis), the softening branch in the global load-displacement curve can be simulated by a displacement-control approach, with the prescribed 
displacement in one degree $[10,11]$ or the prescribed incremental displacement norm $[12,13]$.

Recent development of structural numerical methods extended limit analysis to strain-softening materials by employing the mathematical programming with equilibrium constraint (MPEC) method [14, 15]. Barrera et al. [8] proposed a modified LMM to determine the peak load that can be applied to a structural system composed of strainsoftening materials. However, the convergence of the solution is not guaranteed. Therefore, the motivation of this paper is to determine the peak load that a system with strainsoftening materials can resist. As the first step in extending the LMM to strain-softening materials, this paper focuses on a classical problem, i.e., the static response of a beam column with nonlinear springs, to demonstrate the potential of the proposed method.

The objective of this paper is to derive FE-based kinematic and static solutions for bounding the load-displacement responses of pile foundations under monotonic loading by adopting the extended linear matching method (LMM). The general procedure of the extended LMM is presented first, and then, a kinematic solution from the variation of potential energy and a static solution by satisfying the material constitutive law are derived. The convergence of the extended LMM method for strain-softening materials is discussed thereafter. A case study is conducted on an actual offshore platform failed in pile foundation overturning in Hurricane Ike [16-18]. The proposed method falls into the general group of determining the load multiplier in global displacement-softening problems in FE analysis. The novel features of the proposed method on the potential energy-based kinematic solution (mimic to the upper bound solution in limit analysis) and the yield criteria-based static solution (mimic to the lower bound solution in limit analysis) for bracketing the exact load-displacement response are discussed.

\section{Description of the Extended LMM Method}

2.1. Simplified Pile-Soil Interaction Model. As shown in Figure 1, a pile is assumed to satisfy the Bernoulli-Euler beam theory and then is discretized into a number of finite elements where two nodes are allocated at each end. Moreover, the interaction between pile-soil is simplified by a set of one-dimensional springs. To limit the scope of this study, only steel pipe is considered and the pile cross-sectional bending moment-thrust-curvature relation is taken following Chen and Han [19] (see Appendix). It is worthwhile to notice that the proposed method in the current study is also applicable to other pile materials as long as the bending moment-thrust-curvature curves can be obtained. The soil resistance is taken to follow the recommendations of API RP2GEO [20], i.e., the $t-z$ (pile shaft) and $q-z$ (pile end) curves acting along the pile vertical axis and $p-y$ curves for providing lateral resistance to the pile. Such uncoupled behavior of soil axial and lateral resistance is consistent with conventional analysis procedures for offshore piles, where the majority of the axial capacity is contributed by the lower portion of the pile and the lateral capacity is developed within top 10-20 diameters of the pile below the mudline $[21,22]$.

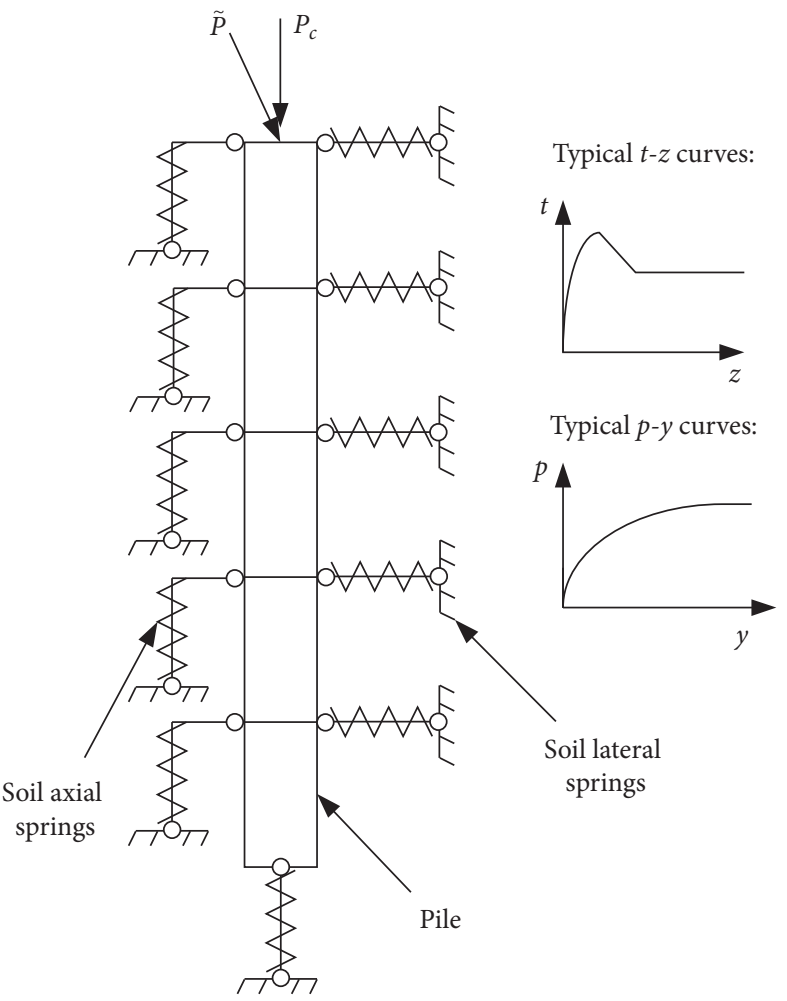

Figure 1: Pile-soil interaction model.

Only a monotonic loading is considered in the current study, and the total loading exerted on the pile can be expressed as $P_{c}+\lambda \widetilde{p}$ similar to Chen et al. [21], where $P_{c}$ is a set of constant gravity loads, $\tilde{p}$ is a unit load vector specifying the direction and location of the proportional loads, and $\lambda$ is a positive load multiplier. Here, it should be noted that $P_{c}+\lambda \tilde{p}$ is a vector. For the above group of loading, the failure of the pile foundation is driven by the proportional load $\lambda \widetilde{p}$. Therefore, it is reasonable to assume that no local elastic unloading occurs under the given loading history, and the generalized stress point is restricted to move along the prescribed constitutive curves (e.g., $t-z$ and $p-y$ curves), such that stress points above or below the curves are not allowed [23]. Consequently, the structural response will be path-independent, which leads to that the current structural response under a given set of loads is independent of the loading history.

2.2. Generalized Stress and Strain. Prager's [19] work-conjugate generalized stress $Q_{j}$ and generalized strain $q_{j}$ are adopted in this study, where the subscript $j$ indicates the $j$ th element in the discretized model. For the convenience of the notation, both pile elements and soil springs are denoted by the structural elements in the following context. Thus, the generalized stresses for a structural element are dependent on the pile cross-sectional axial force, the cross-sectional bending moment, and the axial and lateral soil resistances. The generalized strains are associated with pile axial deformation, bending curvature, and axial and lateral soil spring deformations. 
2.3. Procedure for Load-Displacement Response. A displacement-control loading is adopted to determine the full load-displacement response of the pile with strain-softening $t-z$ and $p-y$ curves. The algorithm for the proposed method is described as follows:

Step 1: during the $k$ th iteration with the load multiplier of $\lambda^{k}$, it is assumed that all the structural elements are linear elastic with the elastic stiffness of $R_{j}^{k}$ for the $j$ th element, where the superscript $k$ and the subscript $j$ indicate the $k$ th iteration and the $j$ th element, respectively. The external displacement field determined by the elastic solution under the given load $P_{c}+\lambda^{k} \tilde{p}$ is given by $\Delta^{k}$. Here, $\Delta^{k}$ is the work-conjugate pair of $P_{c}+$ $\lambda^{k} \tilde{p}$ and is defined at the same reference point as $P_{c}+\lambda^{k} \tilde{p}$, e.g., the pile head. The corresponding internal generalized stresses and strains are, respectively, given by $\bar{Q}_{j}^{k}$ and $\bar{q}_{j}^{k}$, where the bar "-” indicates that the results are from the linear-elastic analysis.

Step 2: the elastic solution is scaled to satisfy the specified displacement criterion, i.e., the analysis is conducted through a displacement-control way that one component of $\Delta^{k}$ is prescribed. Thus, a scaling factor $\mu^{k}$ is determined such that one component of $\mu^{k} \Delta^{k}$ satisfies the prescribed displacement (e.g., the vertical component of $\mu^{k} \Delta^{k}$ equals the prescribed vertical displacement). The corresponding generalized stresses and strains are then given by $\mu^{k} \bar{Q} s_{j}^{k}$ and $\mu^{k} \bar{q}_{j}^{k}$, respectively, based on the linear elasticity. Since $\mu^{k} \bar{Q}_{j}^{k}$ and $\mu^{k} \bar{q}_{j}^{k}$ may not satisfy the prescribed constitutive relation of the structural elements, a new load multiplier is obtained from equation (1) when geometric nonlinearities are excluded:

$$
\left(\lambda_{\text {kin }}^{k+1} \tilde{p}+P_{c}\right) \cdot\left(\mu^{k} \Delta^{k}\right)=\sum_{\text {all elements }} Q_{p j}^{k} \cdot\left(\mu^{k} \bar{q}_{j}^{k}\right),
$$

where the subscript "kin" indicates that the solution is kinematic based on the variation of the potential energy, $\lambda_{\text {kin }}$ is the kinematic load multiplier, and $Q_{p j}^{k}$ is the generalized stress obtained from the constitutive curve corresponding to the generalized strain $\mu^{k} \bar{q}_{j}^{k}$, as shown in Figure 2. The subscript " $p$ " is used to indicate that the generalized stress is on the constitutive curve throughout this paper. The derivation of equation (1) will be given in the next section.

Step 3: by imposing the constitutive laws that all the stress points corresponding to the exact solution can never pass beyond the constitutive curves, a simple approximation is used to scale the elastic solution to obtain a static load multiplier as follows:

$$
\begin{aligned}
\lambda_{s t}^{(k+1)} & =\zeta \lambda_{\text {kin }}^{k}, \\
\zeta & =\min \left[\min _{\text {all elements }}\left(\left|\frac{Q_{p j}^{k}}{\mu^{k} \bar{Q}_{j}^{k}}\right|\right) 1\right],
\end{aligned}
$$

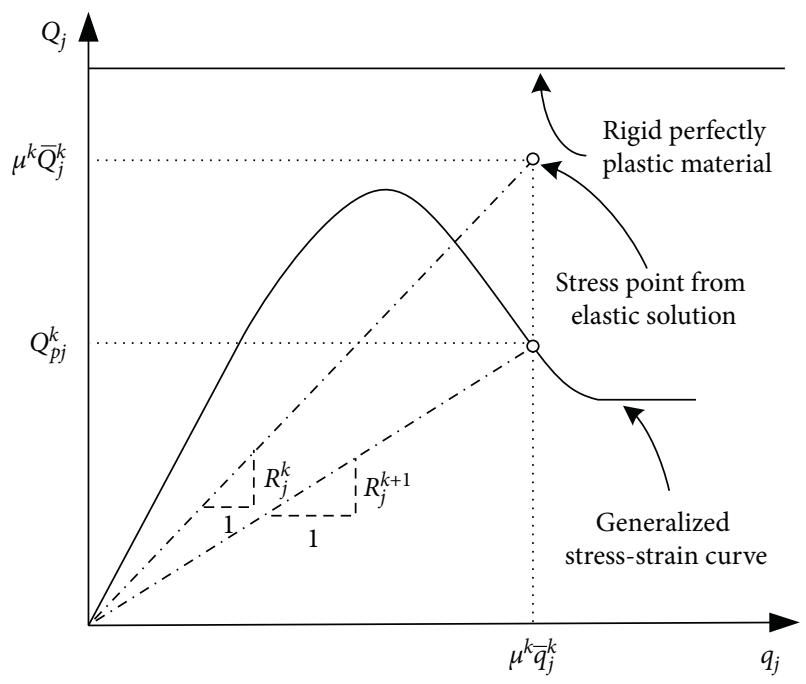

FIGURE 2: Schematic representation of the linear matching process.

where the subscript "st" indicates that the solution is static and $\lambda_{s t}$ is termed the static load multiplier.

Step 4: the stiffness of each element is updated based on the generalized strain $\mu^{k} \bar{q}_{j}^{k}$ (corresponding to the displacement field $\mu^{k} \Delta^{k}$ ) by a linear matching process as follows:

$$
R_{j}^{k+1}=\left|\frac{Q_{p j}^{k}}{\mu^{k} \bar{q}_{j}^{k}}\right|=\left|\frac{Q_{p j}^{k}}{\mu^{k} \bar{Q}_{j}^{k}}\right| R_{j}^{k} .
$$

Let $\lambda^{k}=\lambda_{\text {kin }}^{(k+1)}$, and a new iteration starts with the updated stiffness $R_{j}^{(k+1)}$. Repeat the whole process until a converged solution is obtained.

Once a converged solution is obtained at the prescribed displacement, the prescribed displacement is increased and the iteration is then repeated to find a converged solution for the new prescribed displacement. The convergence is obtained when the kinematic solution equals the static solutions yielding the exact solution. The kinematic solution approaches the exact solution from the upper side, while the static solution approaches the exact solution from the lower side. Thus, the load-displacement response of the system can be determined accordingly. To start the iteration at a specific prescribed displacement, an arbitrary load multiplier $\lambda^{0}>0$ can be chosen. The final solution is independent of $\lambda^{0}$ since $\lambda^{0}$ only provides an initial solution to be scaled and the analysis is controlled by the displacement criterion.

The proposed procedure retains the general characteristics of the LMM presented by Barrera et al. [8], with the extension of the LMM to satisfy the generalized stress-strain relation giving the full load-displacement response of a pile foundation, from which the peak capacity of the pile foundation can be evaluated from a displacement-control way. This full analysis loses the merit of the direct searching for the peak capacity, but gives the full load-displacement response as well as the peak capacity. Therefore, the proposed method provides an alternative for solving pile-soil 
interaction problems when compared to the conventional methods.

2.4. Discussion on Kinematic and Static Solutions. Without geometric nonlinearities, the kinematic solution in equation (1) is obtained from the necessary condition for an exact solution: the first variation of the system potential energy is zero. At the $k^{\text {th }}$ iteration, when the scaling factor $\mu^{k}$ and the associated internal displacement $\mu^{k} \bar{q}_{j}^{k}$ have been determined, the potential energy of the system $U^{k}$ is given as follows:

$$
U^{k}=\sum_{\text {all elements }}\left[\int_{0}^{\mu^{k} \bar{q}_{j}^{k}} Q_{p j}\left(\bar{q}_{j}^{k}\right) \mathrm{d} \bar{q}_{j}^{k}\right]-\left(P_{c}+\lambda_{\mathrm{kin}}^{k} \tilde{p}\right) \cdot\left(\mu^{k} \Delta^{k}\right),
$$

where $Q_{p j}\left(\bar{q}_{j}^{k}\right)$ is the generalized stress corresponding to the generalized strain $\bar{q}_{j}^{k}$ from the generalized stress-strain relation, $\int_{0}^{\mu^{k}} \bar{q}_{j}^{k} Q_{p j}\left(\bar{q}_{j}^{k}\right) \mathrm{d} \bar{q}_{j}^{k}$ is the strain energy of the $j^{t h}$ element at $k^{\text {th }}$ iteration, and $\lambda_{\text {kin }}^{(k+1)}$ is determined by setting $\delta U^{k}=0$ as follows:

$$
\delta U^{k}=\sum_{\text {all elements }} Q_{p j}\left(\mu^{k} \bar{q}_{j}^{k}\right) \delta \bar{q}_{j}^{k}-\left(P_{c}+\lambda_{\text {kin }}^{(k+1)} \tilde{p}\right) \cdot \mu^{k} \delta \Delta^{k}=0,
$$

where $\delta \Delta^{k}$ and $\delta \bar{q}_{j}^{k}$ are the arbitrary pair of external displacement and internal generalized strain, respectively since $\Delta^{k}$ and $\bar{q}_{j}^{k}$ are determined by the linear-elastic analysis and must be kinematically admissible. Thus, $\Delta^{k}$ and $\bar{q}_{j}^{k}$ can be related by a compatible matrix $B$ such that $\bar{q}^{k}=B \Delta^{k}$. Hence, equation (5) can be manipulated to equation (6), from which equation (1) is obtained:

$$
\delta U^{k}=\delta\left[\sum_{\text {all elements }} Q_{p j}\left(\mu^{k} \bar{q}_{j}^{k}\right) \bar{q}_{j}^{k}-\left(P_{c}+\lambda_{\text {kin }}^{(k+1)} \tilde{p}\right) \cdot \mu^{k} \Delta^{k}\right]=0 .
$$

For the static solution, the linear scaling in equations (2a) and (2b) may not necessarily bring all the stress points below the constitutive curve for a concave-down constitutive curve in the prepeak zone. Besides, the constant load $\mathbf{P}_{\mathbf{c}}$ is scaled to be $\zeta P_{c}$, and the original problem has been changed unless $\zeta=1.0$; however, the linear scaling for the static solution does not affect the convergence as it will be discussed below.

For rigid perfectly plastic materials (see Figure 2), $Q_{p j}^{k}$ is independent of $\bar{q}_{j}^{k}$. In this case, the left-hand side of equation (1) gives the input work to the pile-soil system, while the right-hand side gives the energy dissipation in the system. Thus, equation (1) gives the upper bound solution in classical limit analysis, and the solution is independent of $\mu^{k}$. Nevertheless, in general, equations (2a) and (2b) does not constitute a lower bound solution to the rigid-perfectlyplastic system as would be expected since the constant load $\mathbf{P}_{\mathbf{c}}$ is scaled to be $\zeta P_{c}$ as discussed before. Thus, the internal generalized scaled stresses $\zeta \bar{Q}_{j}^{k}$ are in equilibrium with the external load $\zeta\left(P_{c}+\lambda_{\text {kin }}^{k} \tilde{p}\right)$. Therefore, equations (2a) and (2b) give a lower bound load multiplier of $\zeta \lambda_{\text {kin }}^{k}$ only when
$P_{c}=0$ or $\zeta=1.0$. The former case corresponds to zero constant gravity loads; the latter case implies that the lower bound solution coincides with the upper bound solution (see equations ( $2 \mathrm{a})$ and $(2 \mathrm{~b})$ with $\zeta=1.0)$, and the exact solution is obtained. Hence, the closeness of the upper bound solution and the static solution indicates the convergence of the solution for rigid perfectly plastic materials.

For the nonlinear or strain-softening materials, the kinematic and static solutions are not strictly the upper and lower bound solutions. Nevertheless, the determination of the kinematic solution has a sound theoretical basis that the first variation of the potential energy needs to be zero for an exact solution. Besides, the static solution in general ensures the constitutive laws and will not be larger than the previous kinematic solution, i.e., $\lambda_{s t}^{(k+1)} \leq \lambda_{\text {kin }}^{k}$ as can be seen from the scaling in equations (2a) and (2b). When $\lambda_{s t}^{(k+1)}=\lambda_{\text {kin }}^{k}$, all the necessary conditions for an exact solution (compatibility, equilibrium, and constitutive laws) are satisfied. Hence, the solution will be exact for a specific FE formation (i.e., a specific mesh field). Therefore, the closeness of the kinematic and static solution also indicates the convergence of the solution for nonlinear or strain-softening materials.

2.5. Geometric Nonlinearity. The geometric nonlinearity for pile foundation commonly refers to the $P-\Delta$ effect that the axial load will cause secondary bending moment on the pile cross section for a laterally deformation pile. In this case, the axial load on the pile cross section needs to be determined in order to incorporate the $P-\Delta$ effect, and equation (1) needs to be modified accordingly. A revised procedure is described as follows:

Step 1:determine the axial load on the pile cross section without considering the $P-\Delta$ effect using the proposed procedure as described in Section 2.3.

Step 2: repeat the procedure as described in Section 2.3 to incorporate the $P-\Delta$ effect with the following two modifications: (i) the elastic solution is obtained under the consideration of the $P-\Delta$ effect, i.e., the element stiffness of a pile cross section needs to subtract the geometric stiffness that depends on the axial load on the pile cross section; (ii) to estimate the kinematic solution, the input energy from the secondary bending moment applied by the external axial load needs to be considered in equation (1) resulting in

$$
\begin{aligned}
& \left(\lambda_{\text {kin }}^{k+1} \tilde{p}+P_{c}\right) \cdot\left(\mu^{k} \Delta^{k}\right)+\sum_{\text {all elements }} M_{s, j} \cdot \phi_{j} \\
& =\sum_{\text {all elements }} Q_{p j}^{k} \cdot\left(\mu^{k} \bar{q}_{j}^{k}\right),
\end{aligned}
$$

where $M_{s, j}$ is the secondary bending moment caused by the axial load at the $j^{\text {th }}$ pile cross section, and $M_{s, j}$ depends on the pile lateral deflection (i.e., $\mu^{k} \Delta^{k}$ ) and the applied axial load; $\phi_{j}$ is the rotation of the $j^{\text {th }}$ pile cross section that can be taken as the difference of the rotation at the two nodes of a cross section. With the above modifications, the $P-\Delta$ effect can be 
incorporated and a convergent solution can be found through iterations.

\section{Convergence Discussion}

From the linear matching process in equation (3), it can be seen that the updated stiffness is the secant stiffness at $\mu^{k} \bar{q}_{j}^{k}$. Therefore, the proposed method bears the similarity to the incremental-secant (or quasi-Newton) method, and a superlinear asymptotic convergence rate is expected [9]. For rigid perfectly plastic materials, it has been proved that the upper bound solution obtained from the linear matching process in equation (3) decreases monotonically with iterations. However, such feature may not exist for strainsoftening materials.

For the current beam-column problems without the $P-\Delta$ effect, if the load multiplier $\lambda$ remains constant during iterations (i.e., the analysis is load-control, and $\mu^{k}$ remains 1.0), the linear matching process reduces the system potential energy with each iteration. This statement remains valid both for strain-hardening and strainsoftening pile materials and soil springs provided that the pile material and soil springs satisfy the second postulate of the classical plasticity theory [24] in the prepeak region in the generalized stress-strain curve, i.e., the constitutive function of the pile material and soil springs is concavedown with respect to the generalized strains nonincreasing with the increase of generalized strains. The proof is provided below.

As shown in Figure 3, the area of the trapezoid $a-b-d-e$ is not less than the area of polygon $a-b-e-c$ because the constitutive curve is concave-down in the prepeak zone. Therefore, the following inequality holds:

$$
\int_{\bar{q}_{j 1}}^{\bar{q}_{j 2}} Q_{p j}\left(\bar{q}_{j}\right) \mathrm{d} \bar{q}_{j} \leq \frac{\bar{q}_{j 2}^{2}-\bar{q}_{j 1}^{2}}{2 \bar{q}_{j 1}} Q_{p j}\left(\bar{q}_{j 1}\right) .
$$

Using the definition in equations (3), equation (8) can be manipulated to equation (9) as follows (note that $\left.\mu^{k}=1.0\right)$ :

$$
\begin{aligned}
\int_{\bar{q}_{j}^{k}}^{\bar{q}_{j}^{k+1}} Q_{p j}\left(\bar{q}_{j}\right) \mathrm{d} \bar{q}_{j} & \leq \frac{\left(\bar{q}_{j}^{k+1}\right)^{2}-\left(\bar{q}_{j}^{k}\right)^{2}}{2 \bar{q}_{j}^{k}} Q_{p j}\left(\bar{q}_{j}^{k}\right) \\
& =R_{j}^{k+1} \frac{\left(\bar{q}_{j}^{k+1}\right)^{2}-\left(\bar{q}_{j}^{k}\right)^{2}}{2} .
\end{aligned}
$$

The principle of minimum potential for the $(k+1)^{\text {th }}$ elastic solution gives

$$
\begin{aligned}
& \sum_{\text {all element }} \frac{1}{2} R_{j}^{(k+1)}\left(q_{j}^{(k+1)}\right)^{2}-\left(P_{c}+\lambda \widetilde{p}\right) \cdot \Delta^{(k+1)} \\
& \leq \sum_{\text {all element }} \frac{1}{2} R_{j}^{(k+1)}\left(q_{j}^{k}\right)^{2}-\left(P_{c}+\lambda \widetilde{p}\right) \cdot \Delta^{k} .
\end{aligned}
$$

Manipulating equation (10) yields

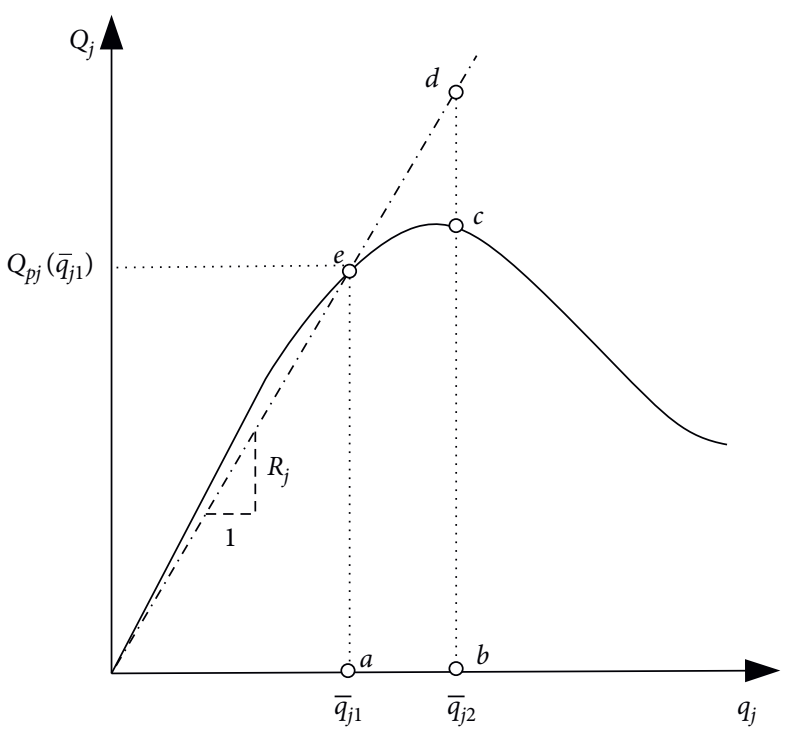

FIGURE 3: Schematic for area calculation.

$\sum_{\text {element }} \frac{1}{2} R_{j}^{(k+1)}\left[\left(q_{j}^{(k+1)}\right)^{2}-\left(q_{j}^{k}\right)^{2}\right] \leq\left(P_{c}+\lambda \widetilde{p}\right) \cdot\left(\Delta^{(k+1)}-\Delta^{k}\right)$.

Combining equations (9) and (11) gives

$$
\sum_{\text {all element }}\left[\int_{\bar{q}_{j}^{k}}^{\bar{q}_{j}^{(k+1)}} Q_{p j}\left(\bar{q}_{j}\right) \mathrm{d} \bar{q}_{j}\right] \leq\left(P_{c}+\lambda \widetilde{p}\right) \cdot\left(\Delta^{(k+1)}-\Delta^{k}\right) \text {. }
$$

Inequality (12) is equivalent to equation (13) as follows:

$$
\begin{aligned}
& \sum_{\text {all element }}\left[\int_{0}^{\bar{q}_{j}^{k+1}} Q_{p j}\left(\bar{q}_{j}\right) \mathrm{d} \bar{q}_{j}\right]-\left(P_{c}+\lambda \widetilde{p}\right) \cdot \Delta^{(k+1)} \\
& \leq \sum_{\text {all element }}\left[\int_{0}^{\bar{q}_{j}^{k}} Q_{p j}\left(\bar{q}_{j}\right) \mathrm{d} \bar{q}_{j}\right]-\left(P_{c}+\lambda \widetilde{p}\right) \cdot \Delta^{k} .
\end{aligned}
$$

The left-hand side of expression 13 is the potential energy of the system at the $(k+1)^{\text {th }}$ iteration, while the righthand side is the potential energy of the system at the $k^{\text {th }}$ iteration. Hence, the matching process reduces the system potential energy monotonically for load-control analyses and ensures the convergence. The equality in equation (13) only occurs when the solution converges such that $\bar{q}_{j}^{(k+1)}=\bar{q}_{j}^{k}$ and $\Delta^{(k+1)}=\Delta^{k}$.

The shortcoming of the load-control analysis is that it is unable to determine the postpeak part of the global loaddisplacement response of a pile foundation. Instead, the displacement-control analysis is preferred to trace the postpeak response. However, the scaling of the linear-elastic solution in the displacement-control analysis to $\mu^{k} \bar{q}_{j}^{k}$ produces a change to the potential energy, and the $P-\Delta$ effect violates the principles of the proof presented above. Therefore, the monotonic reduction of the potential energy with iterations has not been approved and may no longer remain valid. Nevertheless, the energy conservation-based 
kinematic solution (i.e., equations (1) and (7)) still preserves a strong convergence characteristic based on the actual implementation of the proposed method as will be presented in the case study in the next section.

\section{Case Study}

4.1. Platform Description. The proposed kinematic and static solutions are used to determine the global load-displacement response of the pile foundation system of an actual 3-leg offshore platform (Platform $X$ ) that failed in Hurricane Ike in the Gulf of Mexico in 2008. Both two-dimensional and three-dimensional upper and lower bound limit analysis techniques were used comprehensively to determine the pile system capacity of Platform $X$ in the literature $[16,17,25]$, and the system reliability technique was adopted to determine the survival/failure probability of the pile system of this platform [26]. The kinematic and static solutions derived from the current study extend the limit analysis and provide additional tools to determine the full load-displacement response of the pile system of Platform $X$. The extended LMM method proposed in the current study is implemented using the programming software MATLAB to analyse response of the pile foundation.

The description of Platform $X$ is presented here as follows [17]. Platform $X$ is a 3-leg jacket platform (tripod) located in the coast of Louisiana in about $110 \mathrm{~m}$ water depth. A plane view of the pile foundation is shown in Figure 4. Platform $X$ failed in Hurricane Ike in 2008 due to the pull-out of the pile under tensile loading. The pile are steel pile, two of which (Piles $B$ and $C$ in Figure 4) are battered with the vertical-to-horizontal ratio of $5: 1$. Pile $A$ is vertical. A well conductor presents just to the north of Pile $A$; however, this well conductor is neglected in the foundation analysis since the well conductor was not connected in the vertical direction with the platform. The yield strength of the pile is $248 \mathrm{MPa}$. The pile diameters, embedment lengths, and wall thickness schedules are shown in Table 1 . The soil at the platform site is mainly clay. The design undrained shear strength and submerged unit weight profiles are shown in Figure 5. The pile cross-section bending moment-thrust-curvature relation is taken from Chen and Han [19], which consists of a linear-elastic portion, a perfectly plastic portion, and a nonlinear transition between the above two portions (see Appendix). The $t-z$ and $p-y$ curves for soil springs are taken following API RP2GEO [20].

4.2. Single Pile Response. Take Pile $A$ as an analysis example. Pile $A$ is discretized into 60 equal length beam elements. The head of Pile $A$ is assumed to be fixed against rotation and subjected to axial and lateral combined loading. The ratio between the axial and lateral loads is $\alpha$, which remains constant during the loading history. Hence, for small values of $\alpha$, a lateral failure mechanism will be expected, while for larges value of $\alpha$, an axial failure will occur.

4.2.1. Single Ultimate Capacity. If the $t-z$ and $p-y$ curves do not exhibit strain-softening and the geometric nonlinearity (i.e., $P-\Delta$ effect) is excluded, the ultimate limit load at the

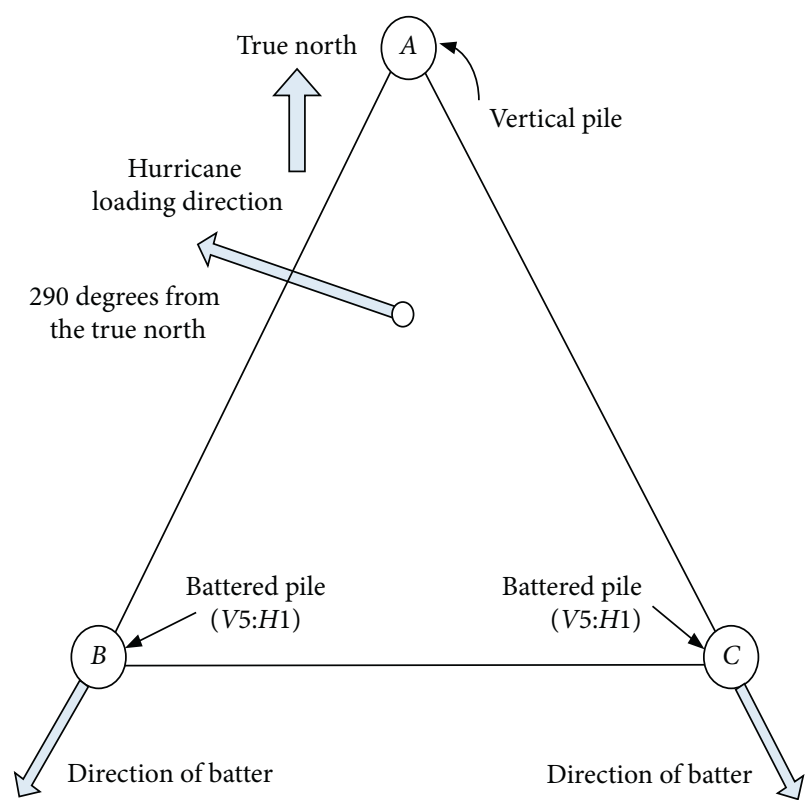

FIgure 4: Pile foundation plan view of Platform $X$.

pile head can be obtained by arbitrarily assigning a large head displacement. Figure 6 shows the variations of the kinematic and static load multipliers (the magnitude of $\widetilde{p}$ is $1 \mathrm{MN}$ ) with the number of iterations for $\alpha=0$ (i.e., purely lateral loading). In this case, the analysis will be exactly the same as the original LMM [6], and the kinematic loading multiplier decreases monotonically with iterations. The kinematic solution converges rapidly initially. Before 10 iterations, the convergence rate is superlinear with efficiency of about 1.36 as expected from the linear matching process (Figure 7). However, the convergence rate decreases with the increase of iterations. The efficiency reduces to about 1.05 after 45 iterations. The decrease of the convergence rate is consistent with the fundamentals of the LMM. The pile is expected to fail by forming two plastic hinges. Thus, the majority of the plastic deformations will concentrate on these two plastic hinges, while the rest portion of the pile will remain rigid from classical limit analysis. For problems involving the large portion of the rigid body, the convergence rate is expected to be slower as the stiffness of a large portion of the body should be updated to an infinite large value (theoretically) in order to simulate a rigid body in classical limit analysis [6]. For this particular case, the static loading multiplier generally increases with iterations. The difference between the two loading multipliers is about $0.06 \%$ after 50 iterations.

Figure 8 shows the result for $\alpha=5$. Both of the kinematic and the static solutions oscillate initially and tend to converge monotonically after 6 iterations. The difference between the two solutions is about $0.5 \%$ after 30 iterations and almost become zeros after 50 iterations. The oscillation of the kinematic solution is due to the adopted yield surface of the steel pile. The cross-sectional ultimate failure surface of the steel pile is shown in equation (A.1). This surface is adopted as the yield surface in the limit analysis. However, this yield surface is categorized as a general yield surface involving the 
TABLE 1: Pile diameter and wall thickness schedule.

\begin{tabular}{lcccc}
\hline \multirow{2}{*}{ Segment no. } & \multicolumn{2}{c}{ Pile $A$} & \multicolumn{2}{c}{ Pile $B$ and $C$} \\
& Penetration below mudline $(\mathrm{m})$ & Wall thickness $(\mathrm{mm})$ & Penetration below mudline (m) & Wall thickness (mm) \\
\hline 5 & 0 to 15.2 & 44.1 & 0 to 16.8 & 37.8 \\
4 & 15.2 to 27.4 & 37.8 & 16.8 to 22.9 & 31.5 \\
3 & 27.4 to 30.5 & 31.5 & 22.9 to 65.5 & 25.2 \\
2 & 30.5 to 79.2 & 25.2 & 65.5 to 67.1 & \\
1 & 79.2 to 80.8 & 31.5 & N/A & \\
\hline
\end{tabular}

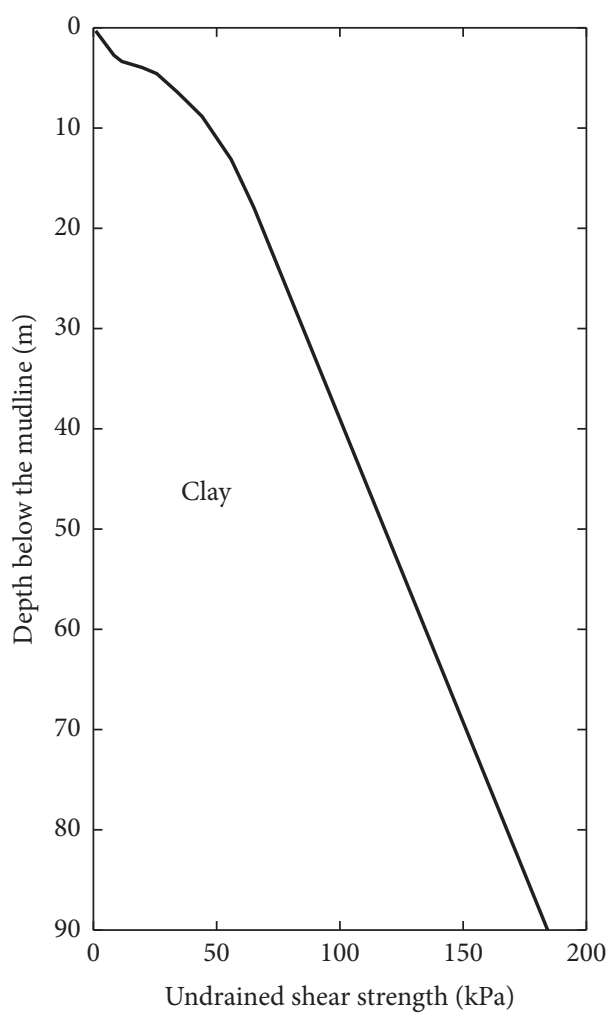

(a)

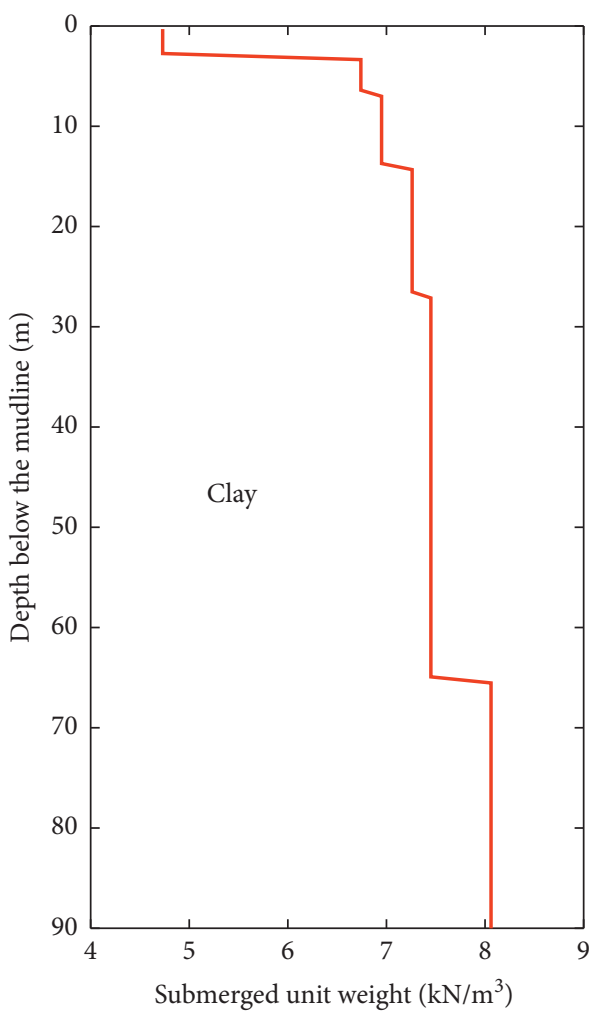

(b)

FIGURE 5: Design undrained shear strength and submerged unit weight profiles.

interactions of axial load and bending moment. As the contour of constant complementary potential passing through a stress point on this yield surface does not enclose the whole yield surface, it does not meet the necessary condition for a monotonic decreasing upper bound solution in the limit analysis using the LMM [7]. Despite of this deficiency, the two solutions converge in all the cases studied in the current study.

For the soil with strain-softening behavior, a detailed load-displacement analysis using the displacement-control approach is adopted. The displacement in one degree of freedom is chosen as an independent variable. The proposed procedure in Section 2.2 is repeated for every required displacement. Thus, the loading multiplier from equation (1) is obtained by ensuring that the first variation of the potential energy is zero. Figure 9 shows the pile head lateral load-displacement response for $\alpha=5$. For Curves 1, 2, and 3, each curve consists of three solutions: the kinematic solution, the static solution, and the mean of the kinematic and the static solutions. In all the cases presented below, iterations stop when the difference between the kinematic and the static solutions is less than $0.5 \%$. As can be seen from the good agreement of three solutions, convergence is achieved for each case presented in Figure 9. For Curve 1, the lateral load increases with the lateral displacement for nonsoftening soils without taking the secondary moment induced by the axial load ( $P-\Delta$ effect) into account. The lateral load is upper limited by the limit load (Curve 4), which only occurs in the limiting case. For softening soils (cyclic $p-y$ curves), as shown by Curve 2, the lateral load increases with the lateral displacement initially and then decreases when some part of soil enters into the postpeak zone in the cyclic $p-y$ curves. The lateral load decreases to the residual load at large displacement, which is shown by Curve 5. Due to the $P-\Delta$ effect, the lateral load may decrease with the lateral displacement due to the secondary moment 


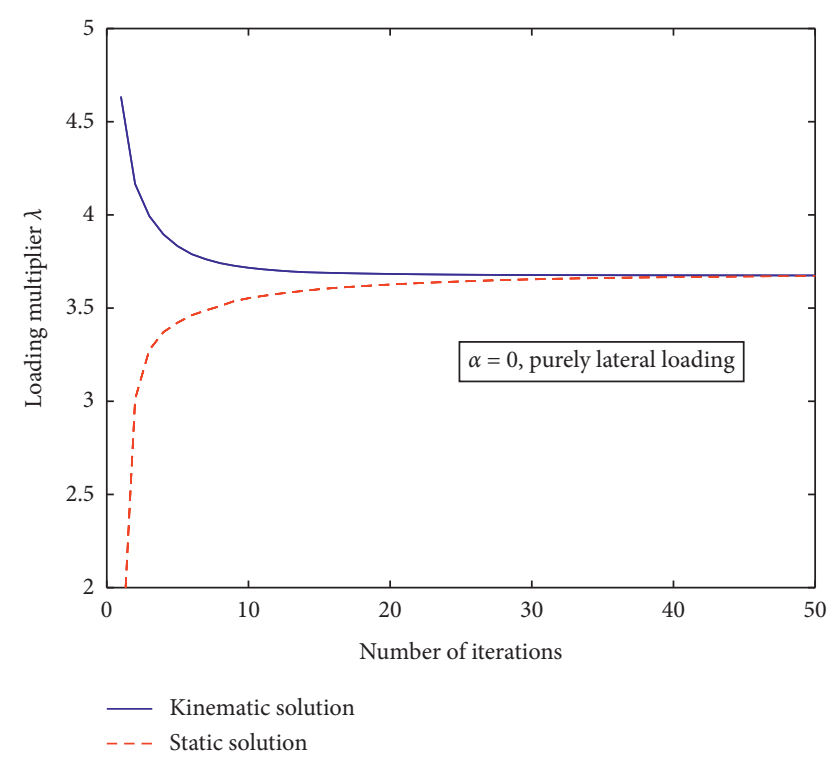

FIgURE 6: Variations of the kinematic and the static loading multipliers for $\alpha=0$.

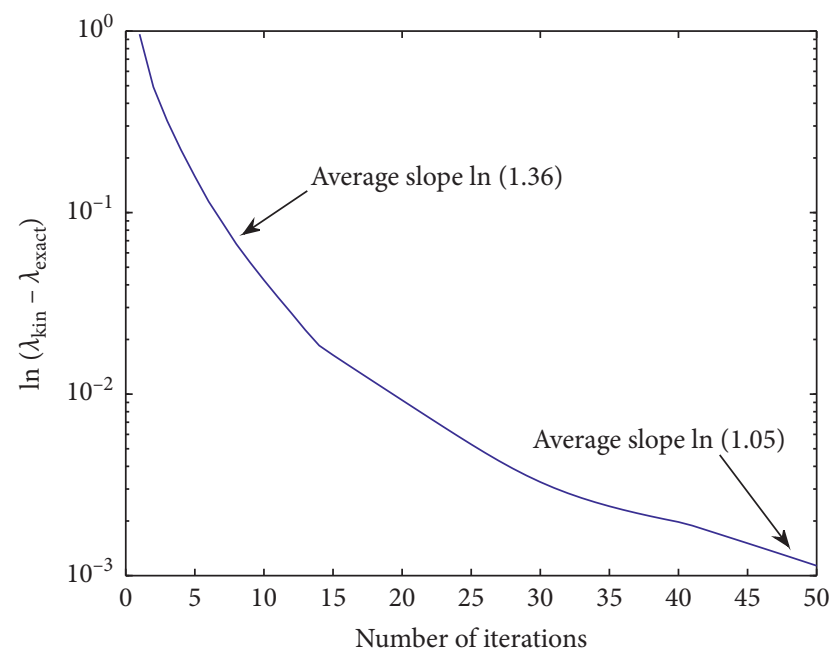

FIgURE 7: Variation of the convergence rate.

induced by the axial load. This effect becomes more significant when the lateral displacement is large, as shown by Curve 3.

Figure 10 shows the axial load-displacement response of a single pile for $\alpha=-15$ (tensile axial load). Similar to the conclusion from Figure 9, the axial load increases with the axial displacement if nonsoftening $t-z$ curves are used, as shown by Curve 1, and the axial load is upper limited by the limit load shown by Curve 3. For softening $t-z$ curves (the residual to the peak value in $t-z$ curves is 0.8 ), the axial load exhibits a peak value and then decreases to a residual value. This effect is well known as the progressive failure of axial loaded offshore piles that the side shear of a pile is not mobilized simultaneously due to the flexibility of that pile. The $P-\Delta$ effect has no effect in this case as the pile is governed by the axial failure mechanism.

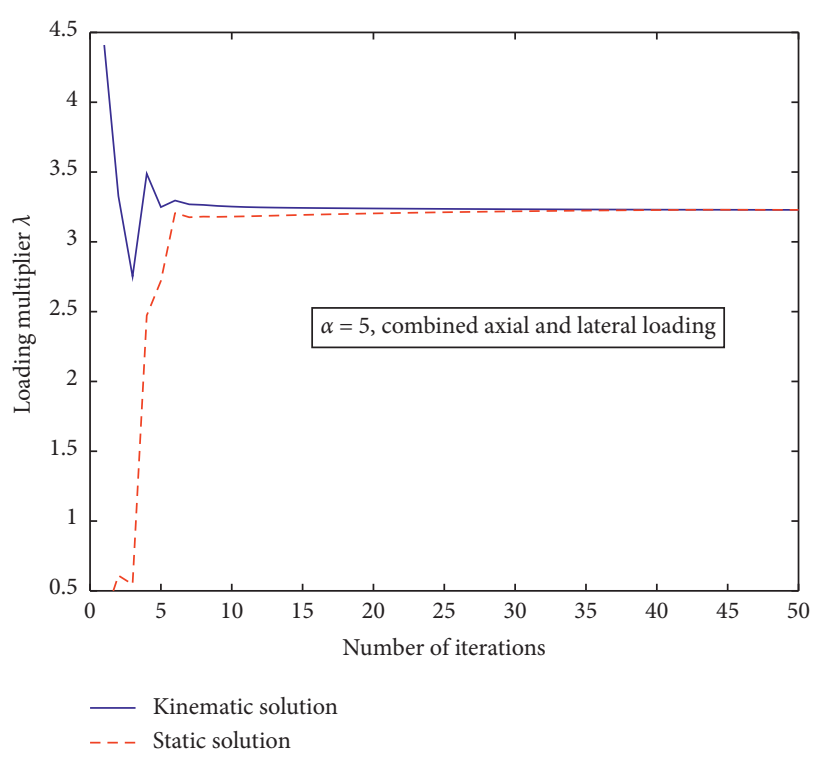

Figure 8: Variations of the kinematic and the static loading multipliers for $\alpha=5$.

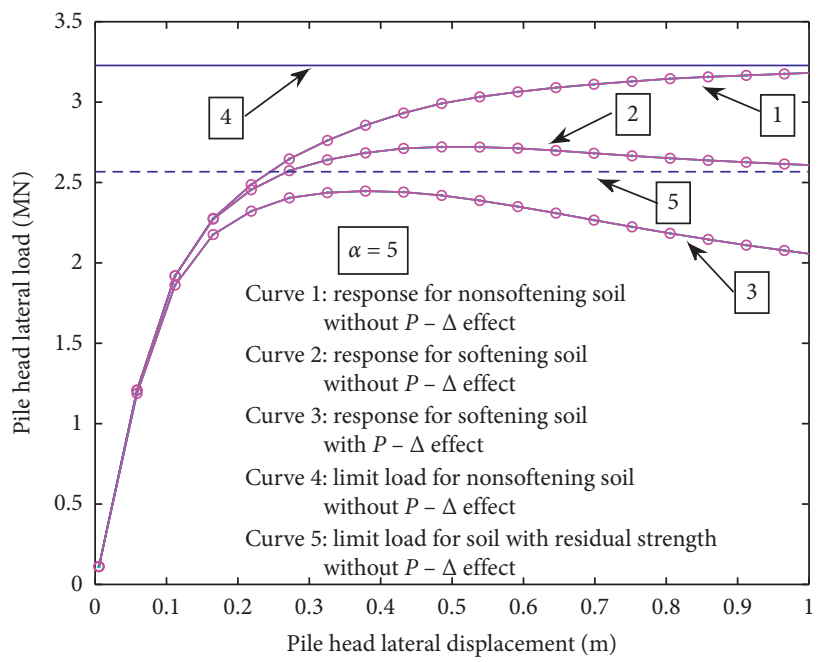

FIgURE 9: Lateral load-displacement response of a single pile.

4.3. Pile System Response. Figure 11 shows the variations of the kinematic and the static horizontal loading multiplier (base load is $1 \mathrm{MN}$ ) with the number of iterations in the limiting case for nonsoftening soils. The kinematic horizontal loading multiplier decreases monotonically with iterations. The monotonically decreasing kinematic solution is consistent with the discussion in Sections 3 and 4.1 because none of the piles yields in this case, and the failure of the jacket is dominated by the pulling out of Pile $C$. Thus, the yield surface of pile steel cross-section does not play in this case, despite each pile is subjected to a combined loading pattern. The static horizontal loading multiplier oscillates initially and increases monotonically to the exact value after 8 iterations.

Figure 12 shows the horizontal load-displacement response of the pile group. As shown, the jacket horizontal 


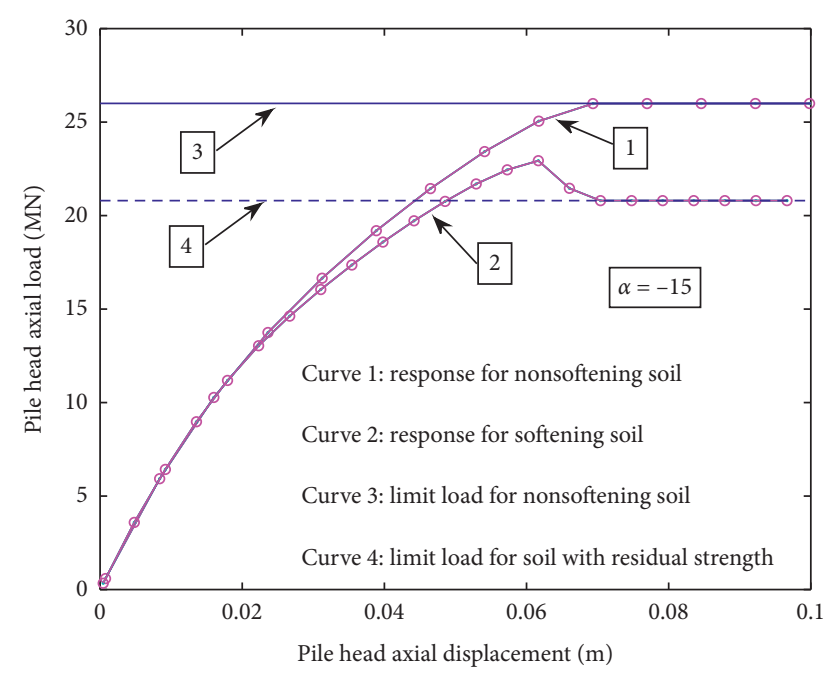

Figure 10: Axial load-displacement response of a single pile.

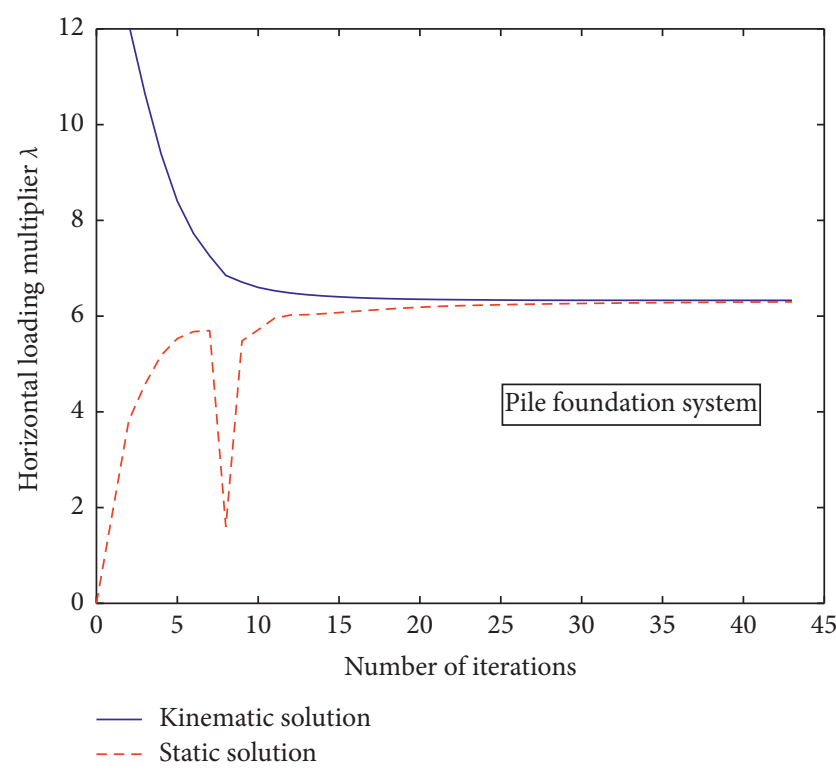

FIGURE 11: Variations of the kinematic and the static loading multipliers for the pile system.

load increases with the jacket displacement for nonsoftening soils (Curve 1). This three-leg platform has little redundancy that the whole platform is approaching failure when Pile $C$ fails. From the plastic limit perspective, additional horizontal load can still be resisted by Piles $A$ and $B$. However, due to the failure of Pile $C$, the system stiffness reduces dramatically that large displacement is required to mobilize any additional resistance after the failure of Pile $C$. This effect can be seen from Figure 12 that the horizontal load-displacement curve almost reaches a plateau when Pile $C$ is pulled out. The horizontal load is upper-bounded by the limiting load shown by Curve 3. For softening $t$ - $z$ curves (the residual to peak value in $t-z$ curves is 0.8 ) and cyclic $p-y$ curves used, the jacket horizontal force reaches a peak and then enters into the postpeak region due to the progressive axial failure of Pile $C$, as can be seen from Curve 2. After the

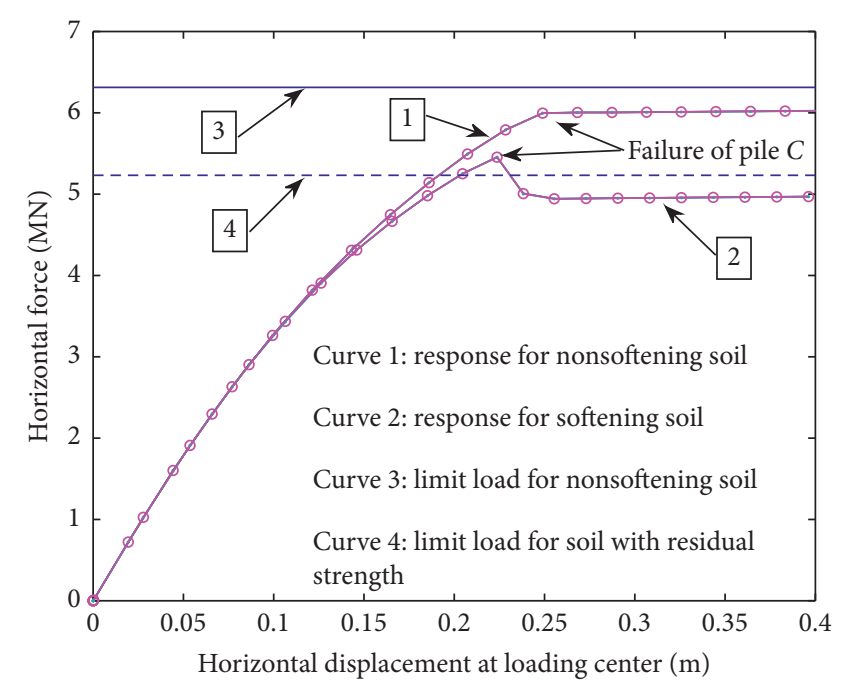

FIgURe 12: Pile system horizontal load-displacement curves.

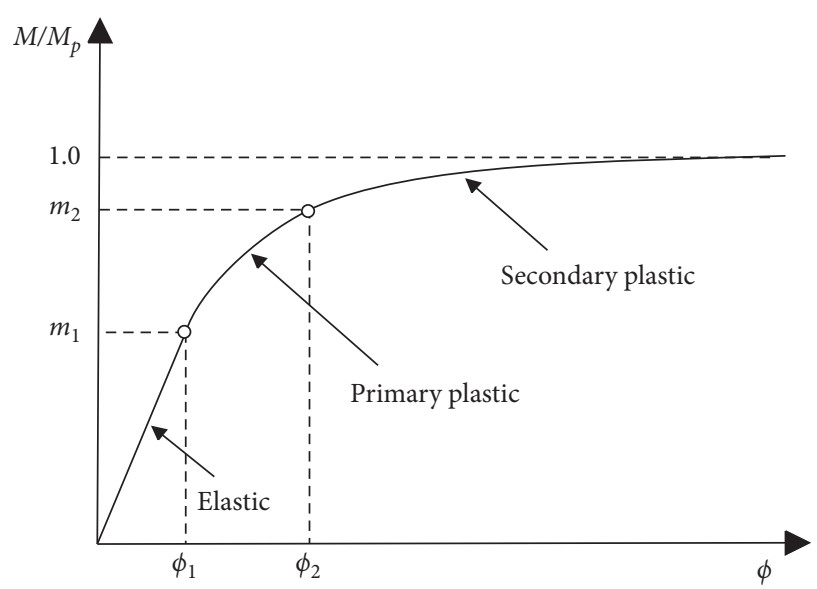

FIGURE 13: Representation of moment-thrust-curvature relation.

failure of Pile $C$, the capacities of Piles $A$ and $B$ are not fully mobilized; the horizontal load drops below the limiting load using the residual strength of soils, as shown by Curve 4 . In the postpeak zone, the horizontal force is expected to increase gradually with displacement in this case, which is upper limited by Curve 4.

\section{Conclusions}

This paper presents kinematic and static solutions for solving the static response of the beam column with nonlinear springs using the linear matching method (LMM). For perfectly plastic materials, the convergence will be guaranteed if the kinematic solution decreases monotonically to the least upper bound solution allowed by the FE formation. For strain-softening materials, although the convergence is not proved directly, the difference between the kinematic and static solutions in each step of iteration can be used as an indicator of the convergence.

The application of the method in offshore pile foundations reveals that a good convergence can be obtained. For 
the steel pile and soil with perfectly plastic materials, the ultimate loads for a single pile and a pile foundation system can be derived in one step by iterations. For soils exhibiting strain-softening, it is convenient to conduct an incremental analysis to determine the peak load. For a single pile, the pile resistance is upper-bounded by the pile limiting load using the peak strength and lower-bounded by the pile limiting load using the residual strength. However, for a pile foundation system, the system load may drop below the pile system limiting load using the residual strength as the capacities of piles are not mobilized simultaneously. The current analysis indicates that the extended LMM proposed in this study has a higher convergence, and further applications in offshore pile foundation analysis can be expected.

\section{Appendix}

Following Chen and Han [19], for elastic perfectly plastic steel, the steel pipe pile cross-sectional ultimate plastic moment resistance $M_{p}$ is given by

$$
M_{p}=M_{p, \max } \cos \left(\frac{\pi}{2} \frac{N}{N_{y}}\right)
$$

where $M_{p, \max }$ is the maximum plastic moment resistance and $N$ and $N_{y}$ are the cross-sectional axial load and axial yield resistance, respectively.

The normalized moment-thrust-curvature relation is given in Figure 13. The parameters $\phi_{1}, \phi_{2}, m_{1}$, and $m_{2}$ depend on the axial load acting on that cross section. The values and related expressions are given in Chen and Han [19] and will not be repeated here. It is assumed that the axial strain is independent of the bending curvature. Thus, the axial strain can be calculated directly from the axial load.

\section{Data Availability}

The data used to support the findings of the study are available from the corresponding author upon request.

\section{Conflicts of Interest}

The authors declare that they have no conflicts of interest.

\section{Acknowledgments}

The authors would like to express the special acknowledgement to Dr. Jinbo Chen, formerly with The University of Texas at Austin, for discussing the case study platform and for sharing his knowledge on the offshore piles and the linear matching method. Without his help, this study would not be completed. Grateful acknowledgment is made to National Natural Science Foundation of China (no. 52078288) to support this research.

\section{References}

[1] A. V. Lyamin and S. W Sloan, "Upper bound limit analysis using linear finite elements and non-linear programming," International Journal of Numerical Analytical Methods in Geomechanics, vol. 26, no. 2, pp. 181-216, 2012.
[2] S. W. Sloan, "Lower bound limit analysis using finite elements and linear programming," International Journal for Numerical and Analytical Methods in Geomechanics, vol. 12, no. 1, pp. 61-77, 1988.

[3] S. W. Sloan, "Upper bound limit analysis using finite elements and linear programming," International Journal for Numerical and Analytical Methods in Geomechanics, vol. 13, no. 3, pp. 263-282, 1989.

[4] D. Weichert and G. Maier, Inelastic Analysis of Structures under Variable Loads: Theory and Engineering Applications, Springer Science \& Business Media, Dordecht, Netherlands, 2001.

[5] D. Fu, Y. Zhang, K. K. Aamodt, and Y. Yan, "A multi-spring model for monopile analysis in soft clays," Marine Structures, vol. 72, Article ID 102768, 2020.

[6] A. R. S. Ponter, P. Fuschi, and M. Engelhardt, "Limit analysis for a general class of yield conditions," European Journal of Mechanics-A: Solids, vol. 19, no. 3, pp. 401-421, 2000.

[7] J.-H. Ri and H.-S. Hong, "A modified algorithm of linear matching method for limit analysis," Archive of Applied Mechanics, vol. 87, no. 8, pp. 1399-1410, 2017.

[8] O. Barrera, A. R. S. Ponter, and A. C. F. Cocks, "Extension of the linear matching method to frame structures made from a material that exhibits softening," European Journal of Mechanics-A: Solids, vol. 30, no. 6, pp. 783-793, 2011.

[9] O. C. Zienkiewicz and R. L. Taylor, The Finite Element Method for Solid and Structural Mechanics, Elsevier, Waltham, MA, USA, 2005.

[10] L. S. Moreira and J. B. M. Sousa, E. Parente, Nonlinear finite element simulation of unbonded prestressed concrete beams," Engineering Structures, vol. 170, pp. 167-177, 2018.

[11] M. Fouaidi, A. Hamdaui, M. Jamal, and B. Braikat, "A high order mesh-free method for buckling and post-buckling analysis of shells," Engineering Analysis with Boundary Elements, vol. 99, pp. 89-99, 2019.

[12] R. D Borst, M. A. Crisfield, J. J. C. Remmers, and C. V. Verhoosel, Nonlinear Finite Element Analysis of Solids and Structures, John Wiley \& Sons, New York, NY USA, 2012.

[13] P. Ho and C. Le, "A stabilized iRBF mesh-free method for quasi-lower bound shakedown analysis of structures," Computers \& Structures, vol. 228, Article ID 106157, 2020.

[14] S. Tangaramvong and F. Tin-Loi, "Simultaneous ultimate load and deformation analysis of strain softening frames under combined stresses," Engineering Structures, vol. 30, pp. 664674, 2008.

[15] R. Ferrari, G. Cocchetti, and E. Rizzi, "Effective iterative algorithm for the limit analysis of truss-frame structures by a kinematic approach," Computers \& Structures, vol. 197, pp. 28-41, 2018.

[16] J. Chen, R. B. Gilbert, F. J. Puskar, and S. Verret, "Case study of offshore pile system failure in Hurricane Ike," Journal of Geotechnological Geoenvironment, vol. 139, no. 10, pp. 16991708, 2013.

[17] J. Chen, Deterministic and probabilistic analyses of offshore pile systems, civil architectural and environmental engineering, PhD Thesis, The University of Texas at Austin, Austin, TX, USA, 2016.

[18] J. Chen, R. B. Gilbert, L. Manuel, and A. Ku, "Comparative assessment of component and system reliability for offshore platform pile foundations," in Proceedings of the international foundations congress \& equipment expo Innovations in Geotechnical Engineering, pp. 371-388, Orlando, FL, USA, June 2018.

[19] W. F. Chen and D. Han, Tubular Members in Offshore Structures, Pitman Publishing, London, UK, 1985. 
[20] Api RP2GEO, API Recommended Practice, Geotechnical and Foundation Design Considerations, American Petroleum Institute, Washington, DC, 2014.

[21] J. Chen, R. B. Gilbert, Y. S. Choo, P. W. Marshall, and J. D. Murff, "Two-dimensional lower bound analysis of offshore pile foundation systems," International Journal of Numerical Analytical Methods, vol. 40, no. 9, pp. 1321-1338, 2016.

[22] N. Gerolymos, S. Giannakos, and V. Drosos, "Generalized failure envelope for laterally loaded piles: analytical formulation, numerical verification and experimental validation," Géotechnique, vol. 70, no. 3, pp. 1-64, 2019.

[23] K. Enakoutsa, D. D. Vescovo, and D. Scerrato, "Combined polarization field gradient and strain field gradient effects in elastic flexoelectric materials," Mathematics and Mechanics of Solids, vol. 22, no. 5, pp. 938-951, 2015.

[24] J. B. Martin, Plasticity: Fundamentals and General Results, MIT press, Cambridge, MA, USA, 1975.

[25] G. B. Gilbert, J. Chen, B. Materek et al., "Comparison of observed and predicted performance for jacket pile foundations in hurricanes," in Proceedings of the Offshore Technology Conference, Offshore Technology Conference-20861-MS, Houston, TX, USA, May 2010.

[26] J. Chen and R. B. Gilbert, "Offshore pile system model biases and reliability," Georisk: Assessment and Management of Risk for Engineered Systems and Geohazards, vol. 11, no. 1, pp. 55-69, 2017. 